\title{
Chromosomal analysis of blastocyst derived from monopronucleated ICSI zygotes: approach by double trophectoderm biopsy
}

\author{
Silvia Mateo ${ }^{1}$, Francesca Vidal ${ }^{2}$, Lluc Coll ${ }^{1}$, Anna Veiga ${ }^{1,3}$, Montserrat Boada $^{1}$ \\ 1 Reproductive Medicine Service, Department of Obstetrics, Gynaecology and Reproduction, Women's Health \\ Dexeus, Barcelona, Spain. \\ ${ }^{2}$ Cell Biology Unit, Faculty of Biosciences, Universitat Autònoma de Barcelona, Barcelona, Spain. \\ ${ }^{3}$ Stem Cell Bank, Centre for Regenerative Medicine, Barcelona, Spain.
}

\begin{abstract}
Objective: This study aims to increase the knowledge about monopronucleated ICSI-derived blastocysts, analyzing trophectoderm biopsies by aCGH and FISH to evaluate their chromosome constitution.

Methods: Fifteen monopronucleated ICSI-derived blastocysts were studied. Double trophectoderm biopsy was performed and analyzed by FISH and aCGH. The blastocysts were classified according to chromosome constitution. Disagreements between the two techniques were assessed.

Results: Results obtained after FISH and aCGH analyses showed the following: $20 \%(3 / 15)$ and $60 \%$ (9/15) diploid females, respectively; $26.7 \%(4 / 15)$ and $26.7 \%$ (4/15) diploid males, respectively; and $53.3 \%$ $(8 / 15)$ and $13.3 \%(2 / 15)$ mosaics, respectively. No mosaic male embryos were found using FISH or aCGH. There were disagreements in $40 \%(6 / 15)$ of the cases due to the higher detection of mosaicism by FISH compared to aCGH.

Conclusions: The combination of FISH and aCGH has been shown to be a suitable approach to increase the knowledge about monopronucleated ICSI-derived embryos. FISH analysis of blastocysts derived from monopronucleated ICSI zygotes enabled us to conclude that aCGH underestimates haploidy. Some diploid embryos diagnosed by aCGH are in fact mosaic. In cases where these embryos would be used for reproductive purposes, extra analysis of parental genome origin is recommended.
\end{abstract}

Keywords: monopronucleated zygote, trophectoderm biopsy, aCGH, FISH

\section{INTRODUCTION}

The finding of a unique pronucleus is evidence that some error has occurred in the fertilization process. The questions are: why does this happen, how do the resulting embryos appear, and can they be considered for reproductive purposes? Many authors contributed to this research to expand the knowledge about monopronucleated zygotes (1PN) (Munné et al., 1993; Staessen et al., 1993; Levron et al., 1995; Sultan et al., 1995; Staessen \& Van Steirteghem, 1997; Otsu et al., 2004; Van Der Heijden et al., 2009; Mateo et al., 2013; 2017; Azevedo et al., 2014; Rosenbusch, 2014).

The genetic composition of monopronucleated ICSI zygotes may have different parental origin, and the mechanisms leading their formation can be diverse:

i) Gynogenetic embryos derived from 1PN ICSI zygotes could be the result of a parthenogenetic activation, without the participation of the paternal genome. This could be due to the extrusion of spermatozoa to the perivitelline space, the absence of decondensation of the paternal nucleus, or premature paternal chromosome condensation (Flaherty et al., 1998). If only one polar body (PB) is present, the embryo would be diploid. When two PB are found, the embryo will be haploid, or diploid if endoreduplication has occurred. ii) Monopronucleated zygotes can also originate androgenetic embryos when there is correct formation of the male pronucleus, avoiding the formation of the female pronucleus. This could be due to the complete extrusion of maternal genome in the second polar body, or due to the maintenance of the meiotic spindle of the oocyte (Azevedo et al., 2014; Kai et al., 2015).

iii) Monopronucleated zygotes with biparental origin could arise from the formation of a unique pronucleus, including maternal and paternal genomes (Levron et al., 1995; Van Der Heijden et al., 2009; Kai et al., 2015). In this case, the union of the maternal and paternal genetic materials could be produced prior to the membrane formation due to the tight proximity of the spermatozoa and the oocyte spindle, or due to the formation of two pronuclei and a subsequent fusion in one pronucleus (Levron et al., 1995; Flaherty et al., 1998; Meseguer, 2016). The finding of an asynchronous pronuclei has also been reported before, and could be another reason for finding a unique pronucleus (Staessen et al., 1993) when time-lapse methodology is not used.

Concerning the possible reproductive use of these embryos, there are different considerations depending on whether they arise from conventional IVF (cIVF) or intracytoplasmic sperm injection (ICSI). While the former is often accepted for clinical use, embryos from 1PN ICSI zygotes are usually discarded due to the reported high incidence of chromosomal abnormalities.

A recently published paper, reporting that some of these zygotes can reach the blastocyst stage, being euploid and resulting in the birth of a healthy child, suggests that they could be used for reproductive purposes in certain cases (Mateo et al., 2017).

A diagnosis of euploidy must be mandatory to consider any 1PN ICSI-derived embryo for transfer. Currently, the Pre-implantation Genetic Screening (PGS) approach used in most centers is addressed to a Comprehensive Chromosomal Screening (CCS) by array Comparative Genome Hybridization (aCGH) in biopsied trophectoderm cells. However, the main limitation of aCGH is its suitability to ascertain the ploidy status of the studied embryos (Gutiérrez-Mateo et al., 2011; Scriven, 2013), and this is an important limitation when there is risk of having haploid embryos in the cohort studied. Fluorescent in situ hybridization (FISH), although being set aside by most groups since the implementation of aCGH, could easily provide information about embryo ploidy.

The objective of this study is to analyze trophectoderm biopsies by aCGH and FISH, to assess to which extend the use of aCGH may lead to an underestimation of haploidy, and to gain knowledge about the chromosome content of embryos coming from 1PN ICSI zygotes.

\section{MATERIAL AND METHODS}

Fifteen blastocysts from monopronucleated (1PN) zygotes with two polar bodies (2PB) obtained after 
ICSI were analyzed. Patients agreed to donate vitrified blastocysts from 1PN 2PB ICSI zygotes, after being informed that these embryos were considered not suitable for reproduction, because of their reported high incidence of chromosomal abnormalities. Patients signed the corresponding written informed consent. In all cases, embryos from 2PN 2PB zygotes were available for transfer. The study was approved by the institutional review board of the center.

ICSI was performed at $40 \mathrm{~h}$ post HCG administration. After ICSI, zygotes were cultured in LifeGlobal total ${ }^{\circledR}$ media (LifeGlobal ${ }^{\circledR}$ ) and were placed in a time-lapse incubator (EmbryoScope ${ }^{\circledR}$-Vitrolife). Images of 5 focal planes were acquired at every 15 minutes. Dynamic monitoring allowed the presence of only a single PN to be confirmed, and either asynchronous 2PN formation or 2PN fusion to be excluded.

Monopronucleated ICSI zygotes were maintained in culture until blastocyst formation. Blastocysts were vitrified and, after being rejected for reproductive purposes, analyzed for research.

\section{Vitrification and warming}

Kitazato media were used for vitrification/warming according to the manufacturer's protocol and cryotop ${ }^{\circledR}$ (Kitazato ${ }^{\circledR}$ ) was used as support. After warming, all blastocysts were cultured in LifeGlobal total ${ }^{\circledR}$ media for 30 minutes.

\section{Blastocyst biopsy}

For zona drilling, the embryos were moved to LifeGlobal Total ${ }^{\circledR}$ w/HEPES. A laser (NaviLase, OCTAX Microscience $\mathrm{GmbH}$ ) attached to a microscope (U-LH100L-3, Olympus ${ }^{\circledR}$ ) was used. Three laser pulses of $1.3 \mathrm{~ms}$ were applied in the zona pellucida of the blastocyst, opposite to the inner cell mass. After zona drilling, the blastocysts were placed in LifeGlobal total ${ }^{\circledR}$ media ( LifeGlobal ${ }^{\circledR}$ ) for at least 8 hours, to facilitate the trophectoderm (TE) herniation for later biopsy. Biopsy of two different TE fragments of each blastocyst were collected by aspiration (between 4 and 16 cells each, depending on the characteristics of the TE).

\section{Chromosomal analysis}

FISH was performed in one of the TE fragments and aCGH analysis in the other.

\section{FISH analysis}

TE cells were washed in phosphate-buffered saline solution (PBS) (6\% human serum albumin) and were fixed on a slide by adding Carnoy's solution (methanolacetic acid; $3: 1$ ). The fixation procedure was performed under an inverted microscope and was adapted to the fragment's characteristics for accurate cell spreading. Biopsies were individually fixed on different slides to avoid cell contamination from other blastocysts. After fixation, the slides were left to dry and they were stored at $-4^{\circ} \mathrm{C}$ until they were processed for FISH analysis.

Since the objective of the FISH analysis was ploidy assessment, the slides were processed for FISH using a commercial probe panel, specific for chromosomes $X, Y$ and 18 (AneuVysion Multicolor DNA Probe Kit, Vysis CEP $18 / X / Y$, Abbott Molecular) according to the manufacturer's protocols. Interphase nuclei were evaluated using an Olympus BX-61 fluorescent microscope (Olympus ${ }^{\circledR}$ ) equipped with specific filters for FITC, Cy3, and Aqua and a multiband pass filter (DAPI/FITC/Texas Red). Overlapped nuclei, metaphase figures and chromatin fragments were discarded from the analysis. Embryos were further classified according to their chromosomal constitution. According to FISH results, the embryos were classified into haploid $(H)$, diploid $(D)$ or mosaic $(M)$, and further gender identified as $f$ (female) or $m$ (male) depending on the results of the cells analyzed. According to this, Df and Dm were those embryos with all the cells analyzed containing two chromosomes 18 and $\mathrm{XX}$ or $\mathrm{XY}$, respectively. Mf and $\mathrm{Mm}$ corresponded to those mosaic embryos with more than one cell line but at least one cell with two copies of chromosome 18 and $\mathrm{XX}$ or $\mathrm{XY}$ respectively. $\mathrm{H}$ were those embryos with all the cells analyzed with one chromosome 18 and one sex chromosome.

\section{aCGH analysis}

TE cells were washed separately in four drops of $10 \mu \mathrm{l}$ of PBS/PVA solution and were transferred to a PCR tube with a drop of $0.1 \mu$ l of PBS solution. The PCR tubes with the samples were stored at $-80^{\circ} \mathrm{C}$ until they were processed. The trophectoderm cells were processed for DNA amplification and aCGH analysis using 24 sure kit and Fluorescent labelling system (Illumina ${ }^{\circledR}$ ), according to the manufacturer's protocols. Hybridization results were processed and analyzed with the BlueFuse software (Illumina ${ }^{\circledR}$ ). The embryos were further classified according to their chromosomal constitution. According to aCGH results, the embryos were classified into diploid (D) or mosaic (M), and further gender identified as $f$ (female) or $m$ (male). According to this classification, Df and Dm were those embryos showing diploid female or diploid male homogeneous chromosomal complements, respectively. Mf and $\mathrm{Mm}$ corresponded to mosaic embryos with diploid female or male cell lines, respectively.

\section{RESULTS}

Fifteen 1PN 2PB-derived blastocysts were successfully thawed (100\% survival rate) and all had re-expanded their blastocoel cavity after $8 \mathrm{~h}$ of culture.

Results from FISH and aCGH analysis and embryo classification are detailed in Tables 1 and 2 respectively.

Concerning FISH results, haploid, diploid female, diploid male and tetraploid cells were found. Diploid cells were found in all the embryos studied. Haploid cell lines were observed in $46.7 \%$ of the embryos (7/15) and tetraploid cells were found in $20 \%$ of the embryos (3/15). No aneuploidy for any of the three chromosomes analyzed was observed (Table 1 ).

According to FISH results, 20\% (3/15) of the embryos were classified as diploid female, $26.7 \%(4 / 15)$ as diploid male and $46.7 \%(7 / 15)$ as mosaic female. One embryo (E12) was classified as mosaic $(6.7 \% ; 1 / 15)$ with coexisting diploid male, diploid female and haploid cell lines. No haploid embryos and no mosaic diploid male embryos were found (Table 1).

Concerning $\mathrm{aCGH}$, the embryos were classified as diploid female in $60 \%(9 / 15), 26.7 \%(4 / 15)$ as diploid male, one mosaic female $(6.7 \%)$ and one mosaic for the sex chromosomes (6.7\%). Aneuploidy was found in $26.7 \%$ of the embryos (4/15). In two diploid male embryos (E6 and E8), the results were consistent with homogeneous aneuploidy for all the cells analyzed. The other two aneuploidy embryos (E4 and E12) were mosaic (Table 2).

Total agreement between FISH and aCGH results was found in $60 \%(9 / 15)$ of the embryos, corresponding to embryos diagnosed by FISH as diploid (E1, E6, E8, E10, E11, E14, E15) and mosaic embryos E4 and E12, the latter with mosaicism for the sex chromosomes. Similar percentages between male and female embryos were observed in embryos diagnosed as diploid, representing $46.7 \%$ of the total embryos (Table 3 ).

There was no agreement in $40 \%$ of the embryos (6/15). Seven mosaic female embryos were detected by FISH and, six of them, were classified as diploid females by aCGH. 
Table 1. Results from FISH analysis, embryo classification and percentage of diploid and haploid cells in 1PN 2PB-derived blastocyst. Df: diploid female; Dm: diploid male; Mf: mosaic female; Mm: mosaic male; M: mosaic for the sex chromosomes

\begin{tabular}{|c|c|c|c|c|}
\hline EMBRYO CODE & $\begin{array}{l}\text { FISH RESULTS } \\
\text { [number of cells] }\end{array}$ & \% DIPLOID CELLS & $\%$ HAPLOID CELLS & FISH CLASSIFCATION \\
\hline $\mathrm{E} 1$ & $\mathrm{XX1818[12]}$ & $100 \%(12 / 12)$ & $0 \%$ & $\mathrm{Df}$ \\
\hline E2 & 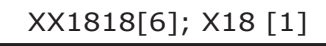 & $85.7 \%(6 / 7)$ & $14.3 \%(1 / 7)$ & Mf \\
\hline E3 & 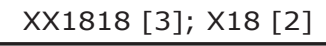 & $60 \%(3 / 5)$ & $40 \%(2 / 5)$ & $\mathrm{Mf}$ \\
\hline E4 & $\begin{array}{c}\text { XX1818 [7]; } \\
X X X X 18181818[1]\end{array}$ & $87.5 \%(7 / 8)$ & $0 \%$ & Mf \\
\hline E5 & 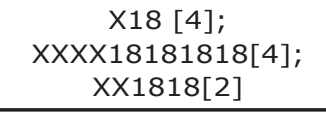 & $20 \%(2 / 10)$ & $40 \%(4 / 10)$ & Mf \\
\hline E6 & XY1818 [5] & $100 \%(5 / 5)$ & $0 \%$ & $\mathrm{Dm}$ \\
\hline E7 & $\begin{array}{l}\text { X18 [5]; XX1818 [1]; } \\
\text { XXXX18181818 [1] }\end{array}$ & $14.3 \%(1 / 7)$ & $71.4 \%(5 / 7)$ & Mf \\
\hline E8 & $\mathrm{XY} 1818[6]$ & $100 \%(6 / 6)$ & $0 \%$ & $\mathrm{Dm}$ \\
\hline E9 & $\mathrm{X} 18[15] ; \mathrm{XX1818[1]}$ & $6.3 \%(1 / 16)$ & $93.8 \%(15 / 16)$ & Mf \\
\hline E10 & $\mathrm{XY1818} \mathrm{[4]}$ & $100 \%(4 / 4)$ & $0 \%$ & $\mathrm{Dm}$ \\
\hline E11 & $\mathrm{XY1818}$ [4] & $100 \%(4 / 4)$ & $0 \%$ & $\mathrm{Dm}$ \\
\hline E12 & $\begin{array}{c}\mathrm{X} 18[7] ; \mathrm{XY} 1818[5] ; \\
\mathrm{XX1818[2]} \\
\end{array}$ & $50 \%(7 / 14)$ & $50 \%(7 / 14)$ & M \\
\hline E13 & 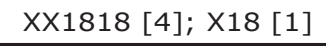 & $80 \%(4 / 5)$ & $20 \%(1 / 5)$ & Mf \\
\hline E14 & XX1818 [5] & $100 \%(5 / 5)$ & $0 \%$ & Df \\
\hline E15 & XX1818 [13] & $100 \%(13 / 13)$ & $0 \%$ & Df \\
\hline
\end{tabular}

\begin{tabular}{|c|c|c|}
\hline $\begin{array}{l}\text { EMBRYO } \\
\text { CODE }\end{array}$ & $\begin{array}{c}\text { aCGH } \\
\text { RESULTS }\end{array}$ & $\begin{array}{c}\text { aCGH } \\
\text { CLASSIFICATION }\end{array}$ \\
\hline E1 & $46, x x$ & Df \\
\hline E2 & $46, x X$ & Df \\
\hline E3 & $46, x X$ & Df \\
\hline E4 & $\begin{array}{c}46, x X / 45, X X \\
(-8)\end{array}$ & Mf \\
\hline E5 & $46, x x$ & $\overline{D f}$ \\
\hline E6 & $47, X Y(+22)$ & $\mathrm{Dm} *$ \\
\hline E7 & $46, x X$ & Df \\
\hline E8 & $46, X Y(-16,+21)$ & Dm* \\
\hline E9 & $46, x x$ & Df \\
\hline E10 & $46, X Y$ & $\mathrm{Dm}$ \\
\hline E11 & $46, X Y$ & $\mathrm{Dm}$ \\
\hline E12 & $\begin{array}{c}46, X Y / 46, X X Y \\
(-6)\end{array}$ & $M$ \\
\hline E13 & $46, x x$ & $\overline{D f}$ \\
\hline E14 & $46, x x$ & Df \\
\hline E15 & $46, x X$ & Df \\
\hline
\end{tabular}

Classification disagreements between the two techniques were due to a higher detection of mosaicism by FISH when compared to aCGH $(53.3 \% ; 8 / 15$ vs. $13.3 \% ; 2 / 15)$. The remaining embryo was classified as mosaic female, an agreement between both techniques (Table 3 ).

In all the embryos analyzed, gender assignment was concordant between the two techniques. Special attention should be paid to embryo E12, which showed mosaicism for the sex chromosomes (Table 3).

\section{DISCUSSION}

There are few papers where monopronucleated ICSI zygotes were cultured until blastocyst stage (Otsu et al., 2004; Mateo et al., 2013; 2017; Yao et al., 2016) and all came from a small study cohort. The reason for the low number of blastocysts included in the present study was the low incidence of monopronucleated ICSI zygotes in the IVF program (3.1\%, unpublished data, Dexeus Women's Health), the restrictive 1PN 2PB assessment using timelapse and the low development potential of 1PN 2PB ICSI zygotes with regard to reaching the blastocyst stage (14.8\%, Mateo et al., 2013; 3.6\%, Yao et al., 2016; 3.4\%, Mateo et al., 2017).

The disagreements found between the techniques used can be explained because a high number of mosaic female embryos detected by FISH were misclassified by aCGH, due to their failure in detecting haploid and tetraploid cells. Even so, it cannot be ruled out that the disagreements were the result of analyzing two different TE fragments; it seems unlikely because the most commonly observed mosaicism involved ploidy alterations. The coexistence 
Table 3. Agreement between classifications obtained after FISH and aCGH analysis of 1PN 2PB-derived blastocysts. H: Haploid; Df: diploid female; Dm: diploid male; Mf: mosaic female; Mm: mosaic male; $\mathrm{M}$ : mosaic for the sex chromosomes

\begin{tabular}{|c|c|c|c|c|c|c|c|c|}
\hline \multicolumn{9}{|c|}{$\mathrm{aCGH}$} \\
\hline & & $\mathrm{H}$ & Df & Dm & Mf & $\mathrm{Mm}$ & M & TOTAL \\
\hline \multirow[t]{7}{*}{ FISH } & $\mathrm{H}$ & 0 & 0 & 0 & 0 & 0 & 0 & 0 \\
\hline & Df & 0 & 3 & 0 & 0 & 0 & 0 & 3 \\
\hline & Dm & 0 & 0 & 4 & 0 & 0 & 0 & 4 \\
\hline & Mf & 0 & 6 & 0 & 1 & 0 & 0 & 7 \\
\hline & $\mathrm{Mm}$ & 0 & 0 & 0 & 0 & 0 & 0 & 0 \\
\hline & $M$ & 0 & 0 & 0 & 0 & 0 & 1 & 1 \\
\hline & TOTAL & 0 & 9 & 4 & 1 & 0 & 1 & $\begin{array}{c}60 \% \\
(9 / 15)\end{array}$ \\
\hline
\end{tabular}

of diploid, haploid and tetraploid cells has been already reported in embryos from monopronucleated zygotes as well as in those which were normally fertilized (Staessen \& Van Steirteghem, 1997; Daphnis et al., 2005). Female embryos showing a high percentage of diploid cells, and few haploid and tetraploid cells could originate from fertilization involving paternal and maternal genomes. The presence of tetraploid cells is a common phenomenon and attributed to the endoreduplication of diploid cells (Daphnis et al., 2005). The presence of few haploid cells could be explained by the formation of binucleate cells with a subsequent cytokinesis (Delhanty et al., 1997). Mosaic female embryos with a high percentage of haploid cells could have originated from oocyte parthenogenetic activation. The embryo initially would be haploid, and later some cells will turn into diploid cells by endoreduplication.

With regards to these findings, embryos in which all the cells analyzed were diploid, those mosaics with diploid/ tetraploid cells and diploid/tetraploid/haploid cells with a low presence of haploid cells $(<50 \%)$ could be considered fertilized; this accounts for $86.7 \%(13 / 15)$. The percentage of diploidy has been shown to be lower when chromosomal assessment is performed in early development (Sultan et al., 1995; Staessen \& Van Steirteghem, 1997; Van Der Heijden et al., 2009; Mateo et al., 2013) but higher, when it is performed in blastocysts (Mateo et al., 2013). The selection against haploid embryos through culturing up to the blastocyst stage could explain these differences (Gras \& Trounson, 1999).

Embryo E12 needs special consideration as coexistent haploid, diploid female and diploid male cells have been observed. These cells could come from the rescue of a triploid XXY embryo, resulting in one diploid male cell line and another haploid cell line, with subsequent diploidization of some haploid cells. Although the rescue of a triploid embryo has been reported after dispermic fertilization, it has not been confirmed after ICSI (Golubovsky, 2003). The origin of this triploid XXY embryo could be the result of either the fecundation of the oocyte by a diploid spermatozoa or by a spermatozoa carrying the $Y$ chromosome together with endoreduplication of the oocyte genome (Rosenbusch, 2008; 2014).

The combination of FISH and aCGH techniques has been a suitable approach to expand the knowledge 1PN 2PB ICSI-derived embryos, and to allow the detection of mosaicism, which would not be detected by applying only one technique. It has been shown that the application of aCGH for analysis may lead to an underestimation of embryos with a high proportion of haploid cells and fully haploid embryos that could compromise the correct development of further pre-implantation stages. Furthermore, it has been demonstrated that FISH approach is not a safe option for the analysis of 1PN 2PBderived embryos for clinical use, as it cannot distinguish between fertilized and non-fertilized diploidized embryos. Even more, FISH analysis where only few chromosomes are analyzed can underestimate the impact of aneuploidy. This is of special importance in 1PN-derived embryos, as high aneuploidy rates have been observed among them (Mateo et al., 2013). Consequently, we recommend an initial analysis with a CCS technique to evaluate the euploidy of $1 \mathrm{PN}$-derived embryos.

In case those embryos would be needed for reproductive purposes, blastocysts diagnosed as diploid males could be considered, as the presence of the $\mathrm{Y}$ chromosome ensures the paternal genome contribution. Despite that, the possibility of an androgenetic origin, with the contribution of only the paternal genome, is not ruled out, although it may seem like a very rare phenomenon (Azevedo et al., 2014; Kai et al., 2015). As aCGH was concordant with FISH in gender assignment, the use of FISH for detecting male embryos will not be necessary. Considering the possible use of diploid female embryos or female mosaics with a high percentage of diploid cells, the use of both FISH and aCGH would not solve the dilemma, as the possible origin by parthenogenesis followed by endoreduplication is not ruled out. The low frequency of uniparental disomy observed in human blastocysts, which is equivalent to those observed in live births, indicates that the presence of uniparental disomy is a rare phenomenon, but it could increase among abnormally fertilized embryos (Gueye et al., 2014; Xu et al., 2015). For better assessment, despite an euploid diagnosis after aCGH has been achieved, other diagnostic methods covering the analysis of parental genome origin should be performed in blastocysts derived from 1PN 2PB ICSI zygotes.

\section{CONCLUSIONS}

The additional use of FISH in the analysis of blastocysts derived from 1PN 2PB ICSI zygotes enabled the conclusion that aCGH underestimates haploidy. Furthermore, some diploid embryos diagnosed by aCGH are, in fact, mosaic. In cases where 1PN 2PB ICSI-derived embryos would be used for reproductive purposes, despite an euploid diagnosis after aCGH has been achieved, an extra analysis of parental genome origin should be performed.

\section{CONFLICT OF INTERESTS}

No conflict of interests has been declared.

\section{Corresponding author:}

\section{Silvia Mateo}

Reproductive Medicine Service

Department of Obstetrics, Gynaecology and Reproduction, Women's Health Dexeus, Barcelona, Spain.

E-mail: silmat@dexeus.com

\section{REFERENCES}

Azevedo AR, Pinho MJ, Silva J, Sá R, Thorsteinsdóttir S, Barros A, Sousa M. Molecular cytogenetics of human single pronucleated zygotes. Reprod Sci. 2014;21:1472-82. PMID: 24717739 DOI: 10.1177/1933719114530185

Daphnis DD, Delhanty JD, Jerkovic S, Geyer J, Craft I, Harper JC. Detailed FISH analysis of day 5 human embryos reveals the mechanisms leading to mosaic aneuploidy. Hum Reprod. 2005;20:129-37. PMID: 15567885 DOI: 10.1093/humrep/deh554 
Delhanty JD, Harper JC, Ao A, Handyside AH, Winston RM. Multicolour FISH detects frequent chromosomal mosaicism and chaotic division in normal preimplantation embryos from fertile patients. Hum Genet. 1997;99:755-60. PMID: 9187668 DOI: $10.1007 / \mathrm{s} 004390050443$

Flaherty SP, Payne D, Matthews CD. Fertilization failures and abnormal fertilization after intracytoplasmic sperm injection. Hum Reprod. 1998;13:155-64. PMID: 9663780 DOI: 10.1093/humrep/13.suppl_1.155

Golubovsky MD. Postzygotic diploidization of triploids as a source of unusual cases of mosaicism, chimerism and twinning. Hum Reprod. 2003;18:236-42. PMID: 12571155 DOI: $10.1093 /$ humrep/deg060

Gras L, Trounson AO. Pregnancy and birth resulting from transfer of a blastocyst observed to have one pronucleus at the time of examination for fertilization. Hum Reprod. 1999;14:1869-71. PMID: 10402407 DOI: $10.1093 /$ humrep/14.7.1869

Gueye NA, Devkota B, Taylor D, Pfundt R, Scott RT Jr, Treff NR. Uniparental disomy in the human blastocyst is exceedingly rare. Fertil Steril. 2014;101:232-6. PMID: 24083874 DOI: 10.1016/j.fertnstert.2013.08.051

Gutiérrez-Mateo C, Colls P, Sánchez-García J, Escudero $T$, Prates $R$, Ketterson $K$, Wells $D$, Munné S. Validation of microarray comparative genomic hybridization for comprehensive chromosome analysis of embryos. Fertil Steril. 2011;95:953-8. PMID: 20971462 DOI: $10.1016 /$ j.fertnstert.2010.09.010

Kai Y, Iwata K, Iba Y, Mio Y. Diagnosis of abnormal human fertilization status based on pronuclear origin and/or centrosome number. J Assist Reprod Genet. 2015;32:158995. PMID: 26395191 DOI: 10.1007/s10815-015-0568-1

Levron J, Munné S, Willadsen S, Rosenwaks Z, Cohen J. Male and female genomes associated in a single pronucleus in human zygotes. Biol Reprod. 1995;52:653-7. PMID: 7756458 DOI: $10.1095 /$ biolreprod52.3.653

Mateo S, Parriego M, Boada M, Vidal F, Coroleu B, Veiga A. In vitro development and chromosome constitution of embryos derived from monopronucleated zygotes after intracytoplasmic sperm injection. Fertil Steril. 2013;99:897-902.e1. PMID: 23246449 DOI: $10.1016 /$ j.fertnstert.2012.11.014

Mateo S, Vidal F, Parriego M, Rodríguez I, Montalvo V, Veiga A, Boada M. Could monopronucleated ICSI zygotes be considered for transfer? Analysis through time-lapse monitoring and PGS. J Assist Reprod Genet. 2017;34:90511. PMID: 28497410 DOI: $10.1007 / s 10815-017-0937-z$

Meseguer M, ed. Time-Lapse Microscopy in In-Vitro Fertilization. Cambridge: Cambridge University Press; 2016. DOI: $10.1017 / 9781316415498$
Munné S, Tang YX, Grifo J, Cohen J. Origin of single pronucleated human zygotes. J Assist Reprod Genet. 1993;10:276-9. PMID: 8130433DOI: 10.1007/BF01204942

Otsu E, Sato A, Nagaki M, Araki Y, Utsunomiya T. Developmental potential and chromosomal constitution of embryos derived from larger single pronuclei of human zygotes used in in vitro fertilization. Fertil Steril. 2004;81:723-4. PMID: 15037434 DOI: $10.1016 /$ j.fertnstert.2003.07.042

Rosenbusch B. The chromosomal constitution of embryos arising from monopronuclear oocytes in programmes of assisted reproduction. Int J Reprod Med. 2014;2014:418198. PMID: 25763399 DOI: 10.1155/2014/418198

Rosenbusch BE. Mechanisms giving rise to triploid zygotes during assisted reproduction. Fertil Steril. 2008;90:49-55. PMID: 17953968 DOI: 10.1016/j.fertnstert.2007.06.031

Scriven PN. The scope, limitations and interpretation of copy number detection in the early embryo using the array CGH technique. Hum Reprod. 2013;28:2-5. PMID: 23054069 DOI: 10.1093/humrep/des366

Staessen C, Janssenswillen C, Devroey P, Van Steirteghem AC. Cytogenetic and morphological observations of single pronucleated human oocytes after in-vitro fertilization. Hum Reprod. 1993;8:221-3. PMID: 8473423 DOI: 10.1093/oxfordjournals.humrep.a138026

Staessen C, Van Steirteghem AC. The chromosomal constitution of embryos developing from abnormally fertilized oocytes after intracytoplasmic sperm injection and conventional in-vitro fertilization. Hum Reprod. 1997;12:321-7. PMID: 9070720 DOI: $10.1093 /$ humrep/12.2.321

Sultan KM, Munné S, Palermo GD, Alikani M, Cohen J. Chromosomal status of uni-pronuclear human zygotes following in-vitro fertilization and intracytoplasmic sperm injection. Hum Reprod. 1995;10:132-6. PMID: 7745042 DOI: $10.1093 /$ humrep/10.1.132

Van der Heijden GW, Van den Berg IM, Baart EB, Derijck AA, Martini E, de Boer P. Parental origin of chromatin in human monopronuclear zygotes revealed by asymmetric histone methylation patterns, differs between IVF and ICSI. Mol Reprod Dev. 2009;76:101-8. PMID: 18481364 DOI: $10.1002 / \mathrm{mrd} .20933$

Xu J, Zhang M, Niu W, Yao G, Sun B, Bao X, Wang L, Du L, Sun Y. Genome-wide uniparental disomy screen in human discarded morphologically abnormal embryos. Sci Rep. 2015;5:12302. PMID: 26194013 DOI: 10.1038/srep12302

Yao G, Xu J, Xin Z, Niu W, Shi S, Jin H, Song W, Wang E, Yang Q, Chen L, Sun Y. Developmental potential of clinically discarded human embryos and associated chromosomal analysis. Sci Rep. 2016;6:23995. PMID: 27045374 DOI: $10.1038 /$ srep23995 